\title{
The Bourdon illusion in haptic space
}

\author{
R. H. DAY \\ Monash University, Clayton, Victoria, Australia
}

\begin{abstract}
A strong Bourdon illusion-the apparent bentness of a straight edge-in the active haptic mode was established in two experiments. Blindfolded subjects clasped the opposite surfaces of an object with the same frontal profile as the visual figure between thumb and forefinger and moved the latter together from end to end across the object. When the two triangular components of the objects were rotated through $180^{\circ}$ so that their bases instead of their apexes were contiguous, the illusion was reversed in direction and reduced by about half, as with the visual illusion. It was concluded that the active haptic Bourdon illusion is basically the same as the visual illu. sion and can be accounted for by a compromise in perception between the orientation of the test surface and that of the object of which it is an integral part.
\end{abstract}

Révész (1934) showed that numerous geometrical illusions, including the Müller-Lyer, Ponzo, and Poggendorff effects, occur when the fingers are either pressed onto or moved over raised or solid forms of the visual stimulus figures. Over (1968) has distinguished between these two forms of stimulation and designated them as passive haptic (pressing) and active haptic (moving fingers). Révész (1934) found that whereas the visual and active haptic illusions were of about the same size, the passive haptic illusions were generally smaller. Haptic geometrical lllusions have since been confirmed by numerous investigators with one or a number of stimulus figures (see Over, 1968). Bean (1938) showed that active haptic illusions occurred with some of a group of totally blind subjects, most of whom had been blind since birth. This result makes it unlikely that the illusions are derived directly from their visual counterparts.

The occurrence of illusions from the same figures appropriately adapted for the visual and haptic modes does not necessarily indicate that the effects are due to the same "extramodal" processes. For example, whereas the visual horizontal-vertical illusion with an L-shaped figure is attributable to meridional anistropies in the visual field (Avery \& Day, 1969), the active haptic effect derives from different velocities of hand movement radially and tangentially to the body (Wong, 1977). In fact, the haptic effect does not occur when the figure is presented in a frontoparallel plane, the usual mode of presentation for the visual figure (Day \& Avery, 1970). Moreover, some visual illusions do not occur in the haptic mode, and others occur in one direction in the visual mode and in the opposite direction in the haptic mode. For example, Hatwell (1960) failed to obtain the Delboeuf illusion in the

The assistance of Richard Hobbs in the construction of apparatus, Fiona Duffy in the collection of data, and Vladimir Kohout, Rosemary Williams, Erica Stecher, and Thais Bassett in the preparation of the manuscript is gratefully acknowledged. Correspondence may be addressed to R. H. Day, Department of Psychology, Monash University, Clayton, Victoria 3168, Australia. active haptic mode, and both Craig (1931) and Parrish (1895) found filled space to be apparently less extensive than unfilled space in the passive haptic mode-rather than more extensive as in the visual.

In formulating an explanation of a perceptual illusion (or a class of illusions), it is of considerable relevance to establish, first, whether the effect occurs in both modes, and, if so, whether the two effects are essentially the same. If they are only "phenotypically" the same, as in the case of the illusions of extent in an L-figure, separate explanations may be indicated. If, on the other hand, they are essentially the same, the explanation must be sufficiently general to account for the effect in both modes.

At least two procedures can be used to establish the commonality or otherwise of the perceptual processes associated with the visual and haptic illusions. The first is to vary a particular stimulus feature and observe the size and direction of the illusion in the two modes. If the effects of the variation turn out to be the same, it is reasonable to conclude that the illusions have processes in common. The second procedure is to induce a decrement in the size of the illusion in one mode by means of repeated presentation of the stimulus figure, the so-called "practice effect." If the reduction of the illusion in one mode transfers to the other, as was found by Rudel and Teuber (1963) for the Müller-Lyer illusion, it can again be concluded that there are processes in common.

The Bourdon illusion occurs in Figure 1. The perfectly straight edge AC appears to be slightly bent about its center B in the same direction as the opposite obtuse angle edge DBE. Day, Mitchell, and Stecher (1989) have shown that the illusion is reversed in direction and reduced in size when the two elongated components of the figure are arranged base to base rather than apex to apex as in Figure 1. The purpose of the two experiments reported here was to establish whether or not the Bourdon illusion occurs in the active haptic mode, and, if so, whether it too is reversed and reduced when the two triangular components of the stimulus object are arranged with their bases contiguous (see Figure 2). Blindfolded subjects 


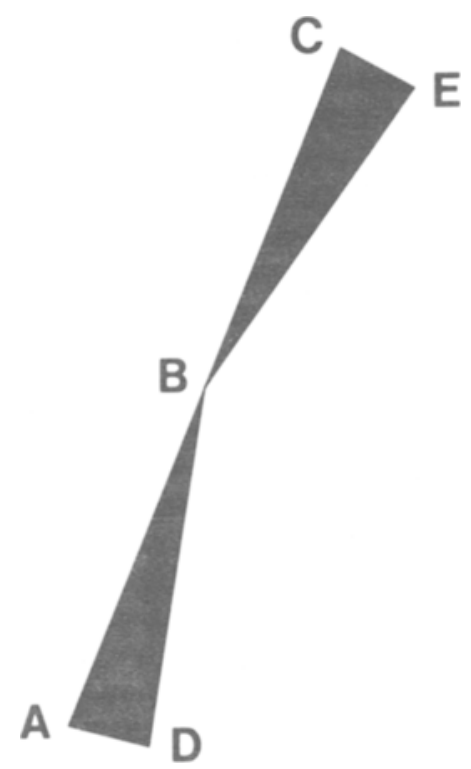

Figure 1. The visual Bourdon illusion. Although the edge $\mathrm{AC}$ is straight, it appears to be bent about its center $B$ in the same direction as the opposite obtuse angle edge DBE.
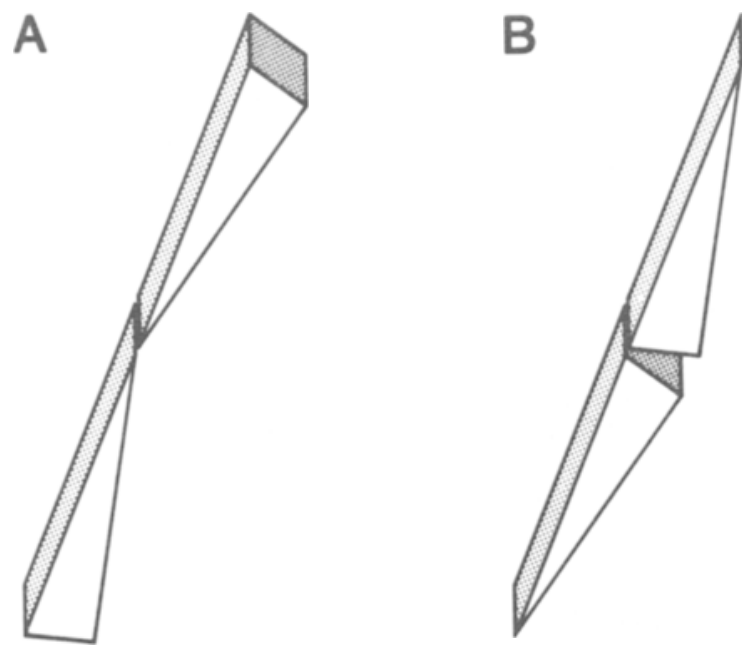

Figure 2. (A) The apex-to-apex stimulus object in Experiment 1. (B) The base-to-base stimulus object in Experiment 2. When the top left test surfaces were flat throughout their lengths, they were oriented at $30^{\circ}$ from the vertical.

moved either the thumb and forefinger together repeatedly across the opposite surfaces of three-dimensional objects with the same shape as the visual figures, or the thumb alone across one surface. The thumb and forefinger moving together across the normally flat (test) and obtuse angle (opposite) surfaces, respectively, constituted the experimental (illusion) condition, and the thumb alone on the test surface provided a control condition. The latter was included, since, as far as is known, the judgment of the flatness or otherwise of surfaces by movement of the thumb has not been investigated hitherto. It is conceivable that any departure from true flatness with thumb and forefinger would occur also with the thumb alone and therefore would not necessarily indicate a haptic illusion analogous to the visual illusion. To ensure reliable and precise measurement, Cornsweet's (1962) double-interleaved staircase procedure was used throughout.

\section{EXPERIMENT 1}

The purpose in Experiment 1 was to establish whether or not the Bourdon illusion occurs in the active haptic mode. Three pilot experiments had shown earlier that when a three-dimensional version of the Bourdon figure was clasped lightly between thumb and forefinger with the former on the flat test surface and the latter on the opposite surface and both then moved from end to end across the object, the test surface was felt to be bent about its center in the same direction as the visual illusion. Since all subjects found this method of haptically judging the test surface natural and easy, it was adopted in the main experiment.

\section{Method}

Subjects. There were 12 subjects, 8 women and 4 men, recruited from among undergraduates, graduate students, and research assistants.

Apparatus and Stimulus objects. The Plexiglas stimulus object (Figure 2) was positioned at the center of a Plexiglas disk $30 \mathrm{~cm}$ in diameter. The disk was positioned in turn, concentrically over a circular cutout $20 \mathrm{~cm}$ in diameter in a $60 \times 60 \mathrm{~cm}$ vertical plywood panel clamped firmly to the edge of a table. The stimulus object was at about shoulder height for a seated subject. The triangular components of the object (Figure $2 \mathrm{~A}$ ) rotated about a point exactly halfway between the slightly separated apexes. This was achieved by mounting the two components of the object on steel plates pivoted at a point coincident with the center of the Plexiglas disk. The components were pushed onto steel pins projecting from the plates and held firmly in place by strong magnets embedded in the Plexiglas and flush with its surface. The arrangement of pins and holes in the components was such that the components could be reversed end to end, so that their bases rather than their apexes were coincident (see Experiment 2). The mechanism for rotating the two plate-mounted components was located on the opposite side of the Plexiglas disk from the subject. The angle to which the components were rotated could be read to the nearest $25^{\circ}$ from a pointer moving around the circumference of a protractor $20 \mathrm{~cm}$ in diameter. When rotated to a particular angle, the components of the object remained firmly in position because of friction in the geared system. When the upper left (test) surface of the stimulus object was flat throughout its length, the pointer was at 0 on the protractor scale. At this setting, the surface was at $30^{\circ}$ to the vertical, an angle which preliminary trials had shown was most natural and comfortable for movement of the thumb and forefinger or thumb alone (see Figure 3) from end to end across the object when the subject was seated slightly to one side at an angle to the plywood panel.

The three-dimensional stimulus object is shown in Figure $2 \mathrm{~A}$. It consisted of two components, each one $62 \mathrm{~mm}$ along the test surface on the left, $61 \mathrm{~mm}$ along the opposite surface on the right, $16 \mathrm{~mm}$ along the end surface at the top and bottom, and $11.5 \mathrm{~mm}$ thick from front to back. The apical angle was $14^{\circ}$, and the end angles were $106.2^{\circ}$ and $59.8^{\circ}$. As will be noted below (see Experiment 2 ), these end angles allowed the components to rotate freely 

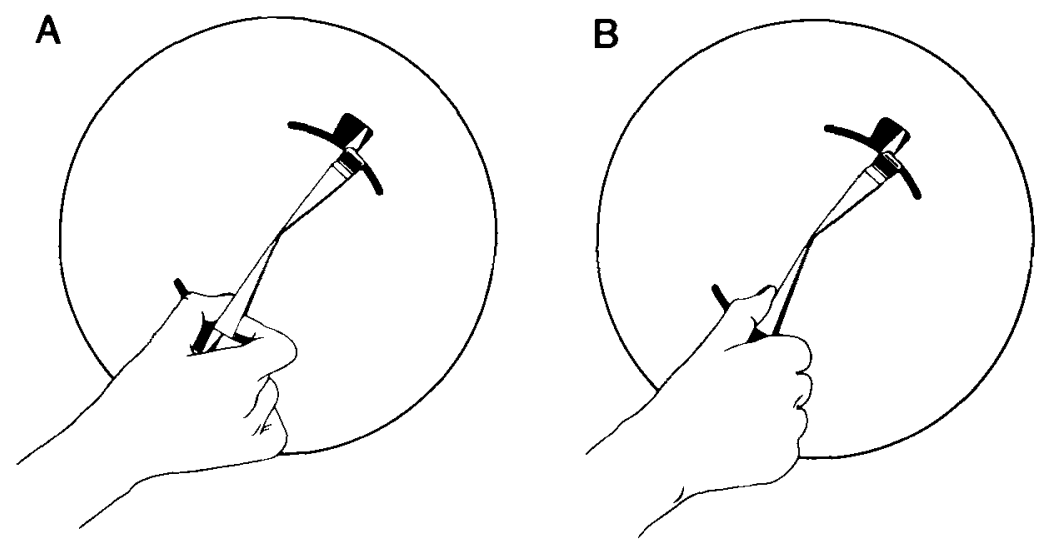

Figure 3. (A) The illusory condition for Experiment 1. The cushion of the thumb was placed on the upper left test surface of the object and the cushion of the forefinger on the lower right obtuse angle surface, and both were moved together from end to end across the object. (B) the control condition for Experiment 1. The thumb alone was placed on the test surface and moved from end to end across it.

when they were arranged base to base. The objects were arranged apex to apex, with a gap of $1 \mathrm{~mm}$ between. The $1-\mathrm{mm}$ gap between the apexes remained constant as the components rotated together about the point exactly halfway between them.

Procedure. The subjects were blindfolded throughout, and at no time before an experimental session did they see the test surface of the object when it was flat. Their task was to indicate the direction in which the surface appeared bent about its center as they moved the thumb alone (control condition) or the thumb and forefinger together (illusion condition) from end to end across the object. These two conditions are depicted in Figure 3.

Two responses were permitted: "up," to indicate that the ends of the test surface were higher and more rightward than the center (the expected direction to compensate for the illusion), and "down," to indicate that they were lower and more leftward (see Figure 3). Responses such as "flat" or "straight" were not acceptable. The subjects were limited to a maximum of four end-to-end movements across the figure, and the response could be made at any time within that limit.

A double-interleaved staircase procedure (Cornsweet, 1962) was used, with $2^{\circ}$ steps in each direction from the zero (flat surface) setting. This step size was selected following preliminary trials to establish what was most appropriate for the haptic task. Following detailed instructions and demonstrations, the subjects moved the cushion of their thumb or those of their thumb and forefinger (see Figure 3) from end to end across the stimulus object and indicated whether the test surface was inflected upward at its ends or downward and rightward. After each response, the hand was taken off the object and rested while the setting of the angle was changed by $2^{\circ}$ in the opposite direction to that indicated by the subject. Thus, if the angle was judged to be up, it was changed by $2^{\circ}$ and vice versa. There were two such staircases, independent and randomly interleaved. This ensured that the subject could not anticipate the direction of change on the basis of the preceding response. The procedure continued until 10 changes from up to down or vice versa had been made. Apparent flatness of the surface was taken to be the mean of the 10 obtuse angles at which these changes in direction occurred. Upward angles were scored as positive, since they were in the direction to compensate for the expected direction of the visual Bourdon illusion, and downward angles were scored as negative. Thus, a mean score of zero indicated that the surface was felt to be flat when it was physically so.

Since, as far as is known, judgment of flatness or bentness by movement of the thumb across a surface has not been previously investigated, it could not be assumed that movement of the thumb alone across the surface would result in a zero or near-zero score. Therefore, a control condition was included in which the thumb alone was used. The control condition (thumb alone) is illustrated in Figure 3B, and the illusion condition (thumb and forefinger) in Figure 3A. Half the subjects undertook the control condition first and the illusion condition second and the other half in the opposite order. The final score for each subject was arrived at by subtracting the control condition score from the illusion condition score. A difference in the same direction as the visual illusion was thus indicated by a positive score-that is, the test surface of the stimulus object would have had to have been inflected farther upward and leftward in the illusion condition than in the control condition to feel flat.

\section{Results and Discussion}

The mean departure from zero (physical flatness) in the control and illusion conditions and the mean differences between them are shown with their standard deviations in Table 1. It can be seen that the mean for the control condition was positive but relatively slight, and that for the illusory condition it was positive and much greater. Whereas the mean score for the illusory condition was significantly different from zero $[t(11)=3.09, p<.01]$, that for the control condition was not $[t(11)=.34$, $p>.10]$. The mean difference score was also significantly different from zero $[t(11)=3.96, p<.005]$. It can be noted that 11 of the 12 difference scores were positive and 1 negative.

These outcomes leave no doubt that a strong illusion of apparent bentness of a flat surface occurs in the same

Table 1

Mean Control, Ilusion, and Difference Scores in Degrees and Their Standard Deviations for Experiments 1 and 2

\begin{tabular}{lcccccccc}
\hline & \multicolumn{2}{c}{ Control } & & \multicolumn{2}{c}{ Illusion } & & \multicolumn{2}{c}{ Difference } \\
\cline { 2 - 3 } & $M$ & $S D$ & & $M$ & $S D$ & & $M$ & $S D$ \\
\hline Experiment 1 & .21 & 2.11 & & 3.65 & 4.90 & & 3.44 & 3.01 \\
Experiment 2 & .68 & 1.64 & & -.84 & 2.96 & -1.52 & 1.20 \\
\hline
\end{tabular}


direction as the visual Bourdon illusion when the thumb is moved across the flat surface of the stimulus object and the forefinger across the opposite obtuse angle surface. It is also clear that the test surface feels more or less flat when the thumb alone is moved over it.

\section{EXPERIMENT 2}

One procedure for establishing whether a haptic illusion is essentially the same as its visual counterpart is to establish whether the former follows the same trend in size and direction as the latter when a particular stimulus feature is varied. Day et al., (1989) showed that when the two triangular components of the conventional Bourdon figure (Figure 1) are each rotated through $180^{\circ}$ so that they are base to base instead of apex to apex, the illusion is reversed in direction and reduced by about half. That is to say, when the triangular components are base to base, the outer ends of the figure, now the apexes, appear to be displaced upward to the left rather than downward to the right as in Figure 1. The purpose in Experiment 2 was to establish whether this reversal and reduction of the illusion occurs when the two components of the haptic object are base to base. Apart from the stimulus object, the experimental conditions were the same as those for Experiment 1 .

\section{Method}

Subjects. There were again 12 subjects, 6 women and 6 men, drawn from the same sources as for Experiment 1. None had participated in Experiment 1.

Apparatus and Stimulus object. The apparatus was the same as before. The stimulus object, with its two components arranged base to base, is shown in Figure 2B. In this configuration, the two test surfaces were again separated by $1 \mathrm{~mm}$ and rotated about the center of the gap. In the base-to-base arrangement, there was an angular gap between the test and opposite surface (Figure 2B). When the test surface was flat throughout its length (i.e., at $0^{\circ}$ ), this angle was $24^{\circ}$ and about $6 \mathrm{~mm}$ across between the opposite surfaces. As pointed out above, this gap occasioned by the angled ends of the components allowed the two components when base to base to rotate in the same manner as in the apex-to-apex arrangement.

Procedure. The instructions, demonstrations, and staircase routines for the control and illusion conditions were the same as those in Experiment 1. With the components arranged base to base, it was expected that the illusion would be opposite in direction to that in Experiment 1. Since the scoring convention was the same as before, the scores were expected to be negative. The order in which the control and illusion conditions were undertaken was again alternated from subject to subject.

\section{Results and Discussion}

The mean scores for the control and illusion conditions and the mean difference scores are shown with their standard deviations in Table 1. The mean for the control condition was small and positive and that for the illusion condition slightly larger but negative. Neither mean proved to be significant $\left[t_{C}(11)=1.42, p>.10 ; t_{I}(11)=1.00\right.$, $p>.10$ ]. However, the mean difference score, which was also negative, was significant $[t(11)=2.17, p<.05]$.
Although these results are statistically less convincing than those for Experiment 1, they nevertheless show that the direction and size of the haptic illusion with the baseto-base arrangement of the object components is opposite in direction to that with the apex-to-apex arrangement and smaller. This outcome requires some comment. When the two components were reversed to form the base-tobase object, an angular gap opened up between them. The question arises as to whether the reduced illusion was due to this gap, which was not present in the apex-to-apex arrangement. The data from the visual illusion renders this unlikely. Wenderoth and O'Connor (1987) have shown that a small gap in the visual figure actually enhances the illusion. In any case, it is now clear that the visual illusion occurs strongly with a right angle arrangement of the components arranged base to base with an even greater angular gap between them (Day et al., 1989). It seems unlikely, therefore, that the reduction in the size of the haptic illusion in the base-to-base object was due to the gap between the components.

\section{GENERAL DISCUSSION}

The experiments described here were intended to answer two questions: Does the Bourdon illusion occur in the active haptic mode, and, if so, is it reversed and reduced like the visual effect when the bases rather than the apexes of the components are contiguous? The answer to the first question is clearly affirmative, and the size of this effect accords closely with that of the visual effect. With a figure of similar dimensions to the haptic object, Rozvany and Day (1980) reported a visual illusion of about $3.8^{\circ}$. The active haptic illusion in Experiment 1 was $3.44^{\circ}$. The answer to the second question is also affirmative. The haptic illusion with the base-to-base object was in the opposite direction to that with the apexto-apex object and less than half the size.

The two main issues for consideration are whether the processes underlying the visual and haptic illusion are essentially the same, and, if so, what the nature of that process is. In regard to the first issue, it has been pointed out in the introduction that there are at least two criteria that serve to indicate whether an illusion occurring in two modes is basically the same. One is an identical trend in the illusion when a particular stimulus is varied. Day et al. (1989) showed that the visual illusion is reversed when the triangular components of the figure are arranged base to base instead of apex to apex. They also showed that the illusion in this arrangement is reduced by about half. The data from Experiment 1 showed that this reversal and reduction occur also in the haptic mode. This suggests that the visual and haptic illusions are derived from a common perceptual process. However, this is not to imply that other mode-specific processes do not interact with the basic process to modulate the size of the illusion in that mode. For example, it is now clear that the Bourdon illusion varies markedly with the orientation of the figure 
(Rozvany \& Day, 1980), which strongly suggests an interaction with the oblique effect (Appelle, 1972; Essock, 1980). This seems not to be so with the haptic illusion. ${ }^{1}$

Walker and Shank (1987) have proposed two possible explanations of the Bourdon illusion. The first involves excitatory and inhibitory interactions between visual feature detectors encoding the orientation of lines and edges. In the second explanation, the illusion is accounted for simply in terms of confusion between the straight and obtuse angle edges of the figure; the straight edge appears slightly bent in the direction of the obtuse angle edge and the latter slightly straightened in the direction of the former. The data obtained by Walker and Shank (1987) failed to differentiate these two explanations clearly.

Given the occurrence of the illusion in the haptic mode and the argument set forth above, an explanation in terms of feature detectors seems less likely. In any case, after a series of experiments on angle illusions in solid and outline figures, Wenderoth and O'Connor (1987) concluded that the Bourdon illusion does not derive directly from neural interactions. They, like Walker and Shank (1987), have therefore attributed it to a failure by subjects to discriminate the test edge from both the obtuse angle edge and the average orientation of the figure itself. However, an explanation in terms of confusion between different orientations runs into a major difficulty. The orientation of the test edge, on the one hand, and that of the figure (or object) and the opposite edge, on the other, are entirely discriminable. There is no sense in which they are mistaken for, or confused with, each other. From this standpoint, an explanation of the illusion in terms of confusion between different orientations is no more than an alternative description of it.

As proposed for the visual Bourdon effect (Day et al., 1989), the haptic effect can be accounted for in terms of a recently proposed general theory of illusory and veridical perception (Day, 1989). The essence of the explanation is that frequently there are numerous cues for a variety of stimulus properties, such as edges, depth, and form. When the cues for a particular property are deliberately contrived to be in conflict, some signaling one state of affairs and others a different one, the outcome in perception will be a compromise between them. This compromise is an outcome of the normal integrative process involving "natural," nonconflicting cues, which leads to veridical perception. In the haptic objects in Figures 2A and $2 \mathrm{~B}$, the cues are the true orientations of the test surfaces and the average orientations of the components of which they are integral parts. These are in conflict: The edges are oriented in one direction, and the components as represented by their bisectors in another. In consequence, there is a compromise between the two orientations, so that the test edges appear to be oriented slightly in the directions of the components. In other words, the illusion represents a perceptual resolution of a conflict be- tween two cues for orientation. The outcome in perception is that the orientations of the test surfaces shift slightly from their true orientations in the direction of the orientations of the components of which they are parts.

\section{REFERENCES}

APPELle, S. (1972). Perception and discrimination as a function of stimulus orientation: The "oblique effect" in man and animals. Psychological Bulletin, 78, 266-278.

AVERY, G. C., \& DAY, R. H. (1969). Basis of the horizontal-vertical illusion. Joumal of Experimental Psychology, 81, 376-380.

BEAN, C. H. (1938). The blind have "optical illusions." Journal of Experimental Psychology, 22, 283-289.

CoRnsweET, T. N. (1962). The staircase method in psychophysics. American Journal of Psychology, 75, 485-491.

Craig, F. E. (1931). Variations in the illusion of filled and unfilled tactual space. American Journal of Psychology, 43, 112-114.

DAY, R. H. (1989). Natural and artificial cues, perceptual compromise and the basis of veridical and illusory perception. In D. Vickers \& P. L. Smith (Eds.), Human information processing: Mechanisms and models (pp. 107-129). London: Elsevier.

DAY, R. H. , AVERY, G. C. (1970). Absence of the horizontal-vertical illusion in haptic space. Journal of Experimental Psychology, 83, 172-173.

Day, R. H., Mitchell, P. J., \& Stecher, E. J. (1989). The Bourdon illusion occurs with aligned, right-angle, and parallel edges. Manuscript submitted for publication.

Essock, E. A. (1980). The oblique effect of stimulus identification considered with respect to two classes of oblique effects. Perception, 9, $37-46$.

Hatwell, Y. (1960). Étude de quelques illusions géométriques tactiles chez les aveugles. L'Année Psychologique, 60, 11-27.

Over, R. (1968). Explanations of geometric illusions. Psychological Bulletin, 70, 545-562.

PARrISH, C. S. (1895). The cutaneous estimation of open and filled space. American Journal of Psychology, 6, 514-520.

RÉvÉsz, G. (1934). System der optischen und haptischen Raumtäuschungen. Zeitschrift für Psychologie, 131, 296-375.

Rozvany, G. I. N., \& DAY, R. H. (1980). Determinants of the Bourdon effect. Perception \& Psychophysics, 28, 39-44.

Rudel, R. G., \& Teuber, H. L. (1963). Decrement of visual and haptic Müller-Lyer illusion on repeated trials: A study of crossmodal transfer. Quarterly Journal of Experimental Psychology, 15, 125-131.

Walker, J. T., \& SHANK, M. D. (1987). The Bourdon illusion in subjective contours. Perception \& Psychophysics, 42, 15-24.

Wenderoth, P., \& O'Connor, T. (1987). Outline- and solid-angle orientation illusions have different determinants. Perception \& Psychophysics, 41, 45-52.

WoNG, T. S. (1977). Dynamic properties of radial and tangential movements as determinants of the haptic horizontal-vertical illusion with an L figure. Journal of Experimental Psychology: Human Perception \& Performance, 3, 151-164.

\section{NOTE}

1. In a subsidiary experiment with 5 subjects, the test surface of the apex-to-apex object (Figure $2 \mathrm{~A}$ ) was oriented horizontally, obliquely left and right at $45^{\circ}$, and vertically. Although a significant illusion occurred in all four orientations, it did not vary significantly as a function of orientation.

(Manuscript received August 14, 1989; revision accepted for publication November 7, 1989.) 